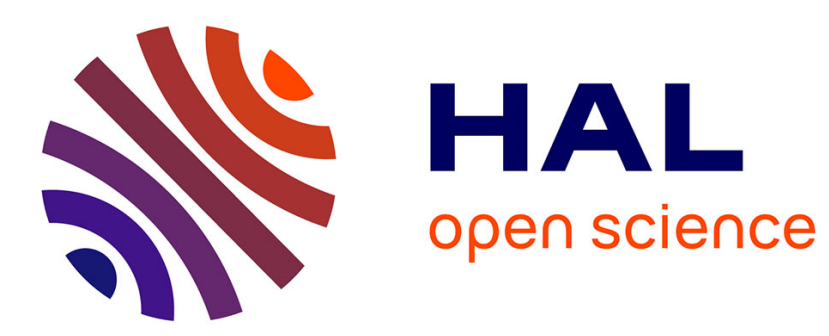

\title{
Lean flame dynamics through a 2D lattice of alkane droplets in air
}

\author{
Colette Nicoli, Bruno Denet, Pierre Haldenwang
}

\section{To cite this version:}

Colette Nicoli, Bruno Denet, Pierre Haldenwang. Lean flame dynamics through a 2D lattice of alkane droplets in air. Combustion Science and Technology, 2014, 186 (2), pp.103-119. hal-00935131

\section{HAL Id: hal-00935131 \\ https://hal.science/hal-00935131}

Submitted on 15 Oct 2015

HAL is a multi-disciplinary open access archive for the deposit and dissemination of scientific research documents, whether they are published or not. The documents may come from teaching and research institutions in France or abroad, or from public or private research centers.
L'archive ouverte pluridisciplinaire HAL, est destinée au dépôt et à la diffusion de documents scientifiques de niveau recherche, publiés ou non, émanant des établissements d'enseignement et de recherche français ou étrangers, des laboratoires publics ou privés. 


\title{
Lean Flame Dynamics through a 2D-Lattice of Alkane Droplets in Air
}

\author{
C. Nicoli ${ }^{1}$, B. Denet ${ }^{2}$, P. Haldenwang ${ }^{1}$ \\ ${ }^{1} \mathrm{M} 2 \mathrm{P} 2,{ }^{2}$ IRPHE \\ Université d'Aix-Marseille / CNRS / Ecole Centrale Marseille; France
}

\begin{abstract}
Flame propagation along a 1-D array or through a 2D-lattice of fuel droplets has long been suggested to schematize spray-flames spreading in a two-phase premixture. The present numerical work considers the fresh aerosol as a system of individual alkane droplets initially located at the nodes of a face-centred 2D-lattice, surrounded by a variable mixture of alkane and air, in which the droplets can move. The main parameters of the study are $s$, the lattice path and $\varphi_{L}$, the liquid loading, which are both varied, whereas $\varphi_{T}$, the overall equivalence ratio, is maintained lean $\left(\varphi_{T}=0.85\right.$ ). Main results are: a) for large lattice path (or when the droplets are large enough), spreading occurs as resulting from two stages : a short time of combustion followed by a long time lag of vaporization; a classical triple flame (with a very short rich wing) spreads around the droplets; b) spray-flame speed decreases as liquid loading increases; c) an elementary model invoking both propagation stages allows us to interpret flame speed as a function of the sole parameter $s \times \varphi_{L}$; d) when the lattice path shortens, the spray-flame exhibits a pattern that continuously goes from this situation to the plane flame front;
\end{abstract}

Keywords : spray-flame; two-phase combustion; heterogeneous combustion; vaporizationdiffusion front; droplet combustion

\section{Nomenclature}
$F(\varphi)$ heat of reaction depending on equivalence ratio
$L e_{i}$ Lewis number of species $i$ in the mixture
$R$ droplet radius
$S$ lattice path
$T_{b}$ adiabatic flame temperature for stoichiometric gaseous mixture
$U_{L} \quad$ adiabatic speed for single-phase premixed flame
$U_{L}^{*}$ adiabatic flame speed for the stoichiometric gaseous mixture
$U_{S F}$ spray-flame speed
$Z$ mixture fraction
Ze Zeldovich number for stoichiometric gaseous mixture
$\phi \quad$ local equivalence ratio
$\varphi_{L} \quad$ liquid equivalence ratio of the fresh spray (liquid loading)
$\varphi_{G}$ gaseous equivalence ratio of the fresh spray
$\varphi_{T} \quad$ overall equivalence ratio of the fresh spray
$\theta$ reduced temperature 

$\Psi_{i}$ reduced mass fraction of specie $i$
$\delta_{L}^{*}$ adiabatic flame thickness for the stoichiometric gas mixture
$\tau_{v} \quad$ characteristic time of vaporization
$\tau_{c}$ characteristic time of combustion

\section{Introduction}

Combustion spreading through a spray is an important concern in a large number of applications, such as diesel engines or gas turbines. On the one hand, the state of the art has clearly identified different regimes of propagation: group combustion of droplets, vaporization controlled propagation, pulsating spray-flames (Umemura and Takamori 2005, Mikami et al. 2006, Suard et al. 2004). Recent microgravity experiments have brought improvements of our understanding of the different mechanisms involved (Pichard et al. 2002., Nunome et al 2002, Nomura et al 2000, Nomura et al 2007). Those well-controlled contributions have been particularly useful in order to provide information on droplet size influence and liquid loading effects on spray vaporization and spray-flame speed promotion. On the other hand, the development of monodisperse droplet generator has led to a number of recent works on flame propagation in an array of droplets under different configurations: 1D array (Kikuchi et al (2005), Mikami et al. (2005), Chen and Lin (2012), Nomura et al. 2013) regular 2D array (Wu and Sirignano 2011) or random configurations (Oyagi et al 2009).

In the paper, we present a set of numerical studies of combustion spreading through a facecentred 2D-lattice of droplets. The simulations are carried out using a simplified chemistry with a global exothermic reaction, and we are particularly interested in the combustion of a globally lean spray, a regime which has not much benefited from numerous studies, although this corresponds to a general trend in the applications. Combustion spreading through this system is here aimed at providing new insights into the problem of spray-flame propagation through a mist composed of droplets, the vaporization time of which is not negligible in comparison with the chemical times. Note furthermore that the lattice of droplets is an initial condition only: when the flame propagates, the droplets are allowed to move. They are driven by two cooperative features: droplet vaporization and thermal expansion of the mixture.

Although the overall spray composition is supposed lean (the overall equivalence ratio is set to $\left.\varphi_{T}=0.85\right)$, the local equivalence ratio $(\varphi)$ can be found rich close to droplets. To study the combustion in such a heterogeneous medium, we have implemented two different chemical schemes, which are both of one-step reaction type. The first scheme is the classical one-step irreversible reaction model, which is known as acceptable in lean pre-mixture. Borrowed from the recent literature (Garrido-Lopez and Sarkar, 2005), the second one introduces a progress variable that allows us to adapt heat release to fresh composition, in order to get satisfactory results on the rich side, too. Their comparison however shows that the spreading features reported here do not markedly depend on those chemical models. Non-dimensioning is carried out thanks to time and length scales of the stoichiometric premixed flame. Four lattice paths have been investigated: $s=1.5, s=3, s=6$ and $s=12$ in units of the latter flame thickness, as well as four different liquid loadings : $\varphi_{L}=0.85, \varphi_{L}=0.65, \varphi_{L}=0.45$ and $\varphi_{L}=0.25$, where $\varphi_{L}$ is the equivalence ratio 
linked to the fuel initially under liquid phase. For each set of these parameters, the droplet size is assessed to keep the overall equivalence ratio fixed to $\varphi_{T}=0.85$.

\section{Modelling the spray flames}

At low pressure, flame thickness often appears large in comparison with droplet interspacing. In the recent years, this allowed us to resort to homogenization for developing an appropriate numerical modelling. In such an approach which also neglects droplet inertia, liquid fuel appears as an additional species subjected to enter into the chemical scheme after a vaporization step. Several spreading regimes have been predicted [Suard et al. 2004], in particular an intrinsic oscillatory regime [Hanai et al. 1998, Atzler et al. 2001] occurring as a Hopf bifurcation. The existence of this regime does not require the implication of differential diffusivity effects [Nicoli et al. 2005 \& 2007].

At moderate and high pressure, spray-flame thickness can no longer be large enough -in comparison with droplet interspacing- to allow any process of homogenization. In such a system, spray-flame tends to be controlled by vaporization, the chemical heat release permitting the vaporization of the droplets one after another. Therefore, spray-flame propagates within a heterogeneous mixture with large droplets, as it is the case of the $n$-decane experiment by Nunome et al. 2002. This is also the configuration of the experiments by Nomura et al. 2000, which was concerned with globally lean mixtures of ethanol and air (with an equivalence ratio about 0.8 ): with droplet interspacing typically of the same size as spray-flame thickness. The aim of the present work is hence to provide a numerical analysis of such lean configurations where the flame "feels" heterogeneities at the droplet length scale. We fix the overall equivalence ratio to 0.85 for the sprays we study. The mist structure is schematized by a face-centred 2D-lattice of alkane droplets in a leaner pre-mixture alkane-air.

On the one hand, the present numerical modeling considers the usual set of conservation laws: mass, momenta, energy and species. Since the accurate chemical schemes for alkane are too complex for efficient numerical simulations, the standard approach searches after a simplified chemical kinetics. As this work is devoted to spray with droplet inter-distance not small in comparison with the characteristic combustion scales (at least, of the same order), our spray-flames will propagate through a medium with varying chemical composition. The simplest manner consists of choosing an irreversible 1-step reaction, the parameters of which are adjusted to mimic the flame dynamics. It is known that the classical one-step Arrhenius law largely overestimates the adiabatic flame temperature on the rich side. Although the calculations presented here concern lean sprayflames, we use two different manners of computing the heat release: either the classical (constant) heat of reaction, or a heat of reaction as a linear function of the fresh gases equivalence ratio (Nicoli and Haldenwang 2010). The second model correctly mimics the characteristics of the premixed single-phase flame (adiabatic flame temperature and flame speed). The use of this model in a heterogeneous mixture is carried out by introducing the mixture fraction as proposed in GarridoLopez and Sarkar (2005) and Fernandez-Tarrazo et al (2006) to suitably adapt heat release to the local composition of the unburnt spray. (see below). The latter point seems, a priori, to be important, since the resulting chemical scheme is able to take account of the heterogeneities in the mixture composition (it must be recalled that flame dynamics depends on the manner the mixture is performed, in the close vicinity of the flame especially). 


\section{Non-dimensioning}

Non-dimensional form of the conservation laws is performed with the use of the theoretical data related the stoichiometric (gaseous) premixed flame, as derived in the theoretical papers by Joulin and Mitani (1981), or Garcia-Ybarra et al (1984). We define the stoichiometric flame temperature, as $T_{b}^{*}$, given by

$$
T_{b}^{*}=T_{u}+\frac{\left(Y_{\mathrm{f}}\right)_{u}^{*} Q}{C_{p} \nu_{f} M_{f}}
$$

Temperature and species mass fractions are handled under the reduced forms

$$
\theta=\left(T-T_{u}\right) /\left(T_{b}^{*}-T_{u}\right) ; \quad \psi_{i}=Y_{i} / Y_{i, u}^{*}
$$

( $i=f$ for the considered alkane and $i=o$ for oxygen)

As for the time and length scales, we define them with the use of $D_{t h, b}^{*}$, the thermal diffusivity coefficient of the burnt gases, and $U_{L}^{*}$, the stoichiometric (single-phase) flame speed, given by

$$
\left(U_{L}^{*}\right)^{2}=\frac{4}{Z e^{3}}\left(\frac{\rho_{b}^{*}}{\rho_{u}^{*}}\right)^{2} \frac{\lambda_{b}}{\rho_{b}^{*} C_{p}}\left[\left(\rho_{b}^{*}\right)^{2} v_{f} M_{f} Y_{o, u}^{*} B_{b}\right] \exp \left(-T_{A} / T_{b}^{*}\right)
$$

This allows us to establish the scalar conservation laws as follows

$$
\begin{gathered}
\frac{\partial \theta}{\partial t}+\bar{V} \cdot \bar{\nabla} \theta=\frac{1}{\rho C_{p}} \operatorname{div}(\lambda \bar{\nabla} \theta)+\mathrm{F}(\varphi) W\left(\rho, \psi_{i}, \theta\right), \\
\frac{\partial \psi_{i}}{\partial t}+\bar{V} \cdot \bar{\nabla} \psi_{i}=\frac{1}{\rho} \operatorname{div}\left(\rho D_{i} \bar{\nabla} \psi_{i}\right)-v_{i} M_{i} W\left(\rho, \psi_{i}, T\right),
\end{gathered}
$$

where the reaction rate is now defined by

$$
\mathrm{W}\left(\rho, \psi_{\mathrm{i}}, \theta\right)=\frac{Z e^{3}}{4}\left(\frac{\rho_{u}^{*}}{\rho_{b}^{*}}\right)^{2} \psi_{f} \psi_{o}\left[\frac{\rho}{\rho_{b}^{*}}\right]^{3} \exp \left[Z e \frac{\theta-1}{1+\gamma(\theta-1)}\right]
$$

with $Z e=T_{A}\left(T_{b}^{*}-T_{u}\right) /\left(T_{b}^{*}\right)^{2}$ and $\gamma=\left(T_{b}^{*}-T_{u}\right) / T_{b}^{*}$.

The overall equivalence ratio of the spray is given by the ratio of the total amount of fuel to the total amount of oxygen in the whole lattice:

$$
\varphi_{T}=\frac{v_{o} M_{o}}{v_{f} M_{f}} \frac{\int_{\text {fesh lattice }} \rho_{f} d v}{\int_{\text {fresh lattice }} \rho_{o} d v},
$$


while the quantity $\varphi_{L}$ [resp. $\varphi_{G}$ ] only takes account of the fuel density under liquid phase [resp. gas phase]. We obviously have $\varphi_{T}=\varphi_{L}+\varphi_{G}$.

\section{Combustion models in a heterogeneous medium}

As mentioned before, the first model -denoted by " $Q_{0} "-$ supposes that the heat of reaction is independent of the local composition. This assumption is only satisfying for the lean side of the composition. On the other hand, we searched after an improvement useful for the rich side, because rich combustion can affect the close vicinity of the droplets. As well-known, combustion on the rich side is characterized by the production of metastable species, the enthalpy of which reduces the flame temperature and therefore the flame speed. A simple analysis of the enthalpy budget at equilibrium and the resulting flame speed has been carried out for several alkanes in (Nicoli and Haldenwang 2010). This approach allowed us to adapt heat of reaction to fresh gas composition. It turned out that the procedure led us to a good agreement with the experimental data on gaseous premixed flame speed. The result took the form of $\mathrm{F}(\varphi)$, a multiplying factor affecting the heat production term in the energy conservation law. $\mathrm{F}(\varphi)$ depends on the equivalence ratio of the fresh gases, linearly as

$$
F(\varphi)=[1-\alpha(\varphi-1)]
$$

where $\alpha$ is a coefficient depending on the considered fuel [refer to a forthcoming paper by the same authors].

Next, we have to leave the concept of homogeneous fresh mixture for considering a medium of variable composition. We now follow the flamelet spirit developed in Garrido-Lopez and Sarkar (2005) (see also Fernandez-Tarrazo et al (2006)). It is well known that mixture fraction is a quantity preserved when crossing a premixed flame, as long as Lewis number is close to unity. As for the opposite case of a diffusion flame, the mixture fraction only results from the transport-diffusive processes and allows us to find the flame positioning. For the triple flame (the intermediate situation), it permits to predict the correct mixing in the fresh gases just in front of the flame. In other words, mixture fraction allows us to assess the mixture composition that the flame burns. More precisely, we compute at any point of the domain

$$
Z=\left(v \psi_{f}-\psi_{o}+r\right) /(v+r)
$$

where $v=24 / 7$ is the stoichiometric oxidizer-alkane mass ratio for a heavy alkane and $r$, the oxidizer proportion in air with $r=\left(\psi_{O}\right)_{i n j} /\left(\psi_{O}+\psi_{N}\right)_{i n j}=0.233$. We hence have $Z \approx 1$ in the droplets (pure fuel), and $Z \approx 0$ in pure air. The dotted line, in figure 1 , represents the mixture fraction for any fresh mixture equivalence ratio. It can be observed that the whole flammability domain corresponds to very small mixture fractions: from $Z \approx 0.04$ for the lean limit $(\varphi=0.5)$ up to $Z \approx 0.09$ at the rich limit ( $\varphi=1.6$ ). In other words, the locus where an alkane spray-flame can propagate occupies a limited area (in $Z$ ) during mixing. To sum up, the method considers $Z$ as a continuous marker of the equivalence ratio in the fresh mixture right before the flame location. 
Furthermore, for the diffusion flame, a classical result leads to linearly link mass fraction of reactants with mixture fraction as follows :

$$
\psi_{f}=\left(\psi_{f}\right)_{\text {injection }} Z \text { and } \psi_{o}=\left(\psi_{o}\right)_{\text {injection }}(1-Z)
$$

Note these hypotheses are consistent with the definition of $Z$. Consequently, in this context, the equivalence ratio that results from a diffusion problem now reads [Garrido-Lopez and Sarkar (2005)]

$$
\varphi=v \psi_{f} / \psi_{o}=v\left(\psi_{f}\right)_{i n j} /(1-Z)\left(\psi_{o}\right)_{i n j}=v Z / r(1-Z)
$$

Hence, the previous adjustable heat of reaction introduced in the equation of energy now reads

$$
\begin{aligned}
& \mathrm{F}(\varphi)=[1-\alpha(v Z / r(1-Z)-1)] \quad \text { if } \quad v Z / r(1-Z) \in[0.5,2] \\
& \text { or } \quad W(\varphi)=0 \quad \text { if } \quad v Z / r(1-Z) \notin[0.5,2] .
\end{aligned}
$$

For the sake of illustrating the efficiency of the adjustment, the homogeneous laminar flame speed of octane-air premixture obtained for $\alpha=0.33$ is plotted in Fig. 1. (Ze being set to 8) and compared with experimental data.

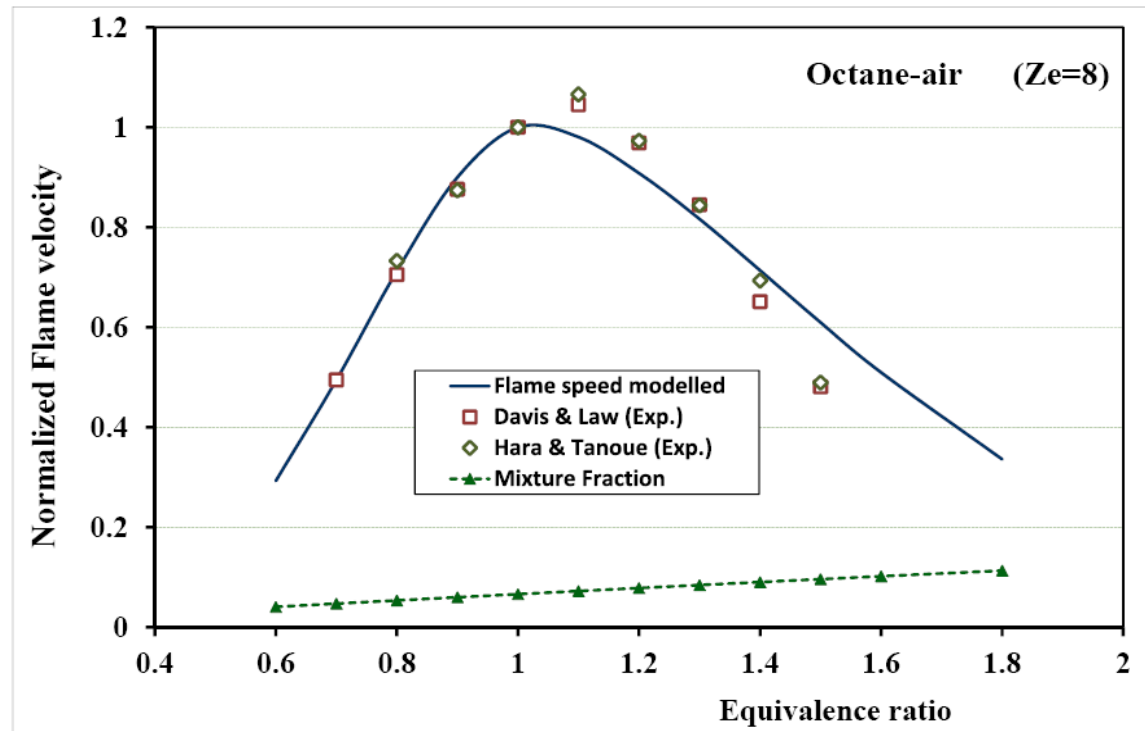

Figure 1: Octane-air flame speed vs. equivalence ratio: comparison between numerical premixed gaseous flame speeds computed with $\alpha=0.33$ and experimental data.

Lastly, as in the conservation laws appears the velocity field $\bar{V}$, the reaction-diffusion system is coupled with the Navier-Stokes equations. The overall numerical scheme considers periodic boundary conditions in the $(y$-) direction transverse to flame propagation, open boundary conditions in the downstream $(x$-) direction, and closed in the $x$-direction upstream from the lattice. The numerical approach of low Mach number type has previously been described in Denet and Haldenwang (1995). In contrast with this previous paper which used constant diffusion coefficients, the present numerical approach uses diffusion coefficients varying with temperature. At low temperature the diffusion coefficient is very small, at high temperature we use the diffusion 
coefficient of an ideal gas, and the formula used interpolates between these two regimes. The result is that diffusion is effectively frozen at low temperature. Let us also note that we use a high Lewis number for the fuel (1.9), which also tends to reduce droplet diffusion. The resulting model is denoted by " $Q(z)$ "

\section{Numerical experiments}

The fuel droplets are positioned at the nodes of the face-centered lattice for a given liquid loading. Since the overall equivalence ratio is set to $\varphi_{T}=0.85$, the droplet radius will only depend on lattice path $s$ and liquid loading $\varphi_{L}$ Four lattice paths have been investigated: $s=1.5, s=3, s=6$ and $s=12$ in units of the stoichiometric premixed flame thickness. Four different liquid loadings have been investigated: $\varphi_{L}=0.85, \varphi_{L}=0.65, \varphi_{L}=0.45$ and $\varphi_{L}=0.25$, where $\varphi_{L}$ is the equivalence ratio linked to the amount of fuel initially under liquid phase. At the open (downstream) end, the temperature field is initiated with the profile of a premixed flame, that allows us to ignite the first droplet of the lattice. To follow the combustion spread, we compute $\langle T\rangle_{y}(x)$, the mean temperature averaged in the periodic (y-)direction. Then, we decide to consider as the flame front, $x_{f}$, the position where $\langle T\rangle_{y}\left(x_{f}\right)=0.5$. This definition can sometimes be misleading because $x_{f}$ is not -by definitiona monotonic function of time: when droplet vaporization is long (in comparison with reaction time), the temperature profile can flatten and $x_{f}$ can seemingly admit a regressing position.
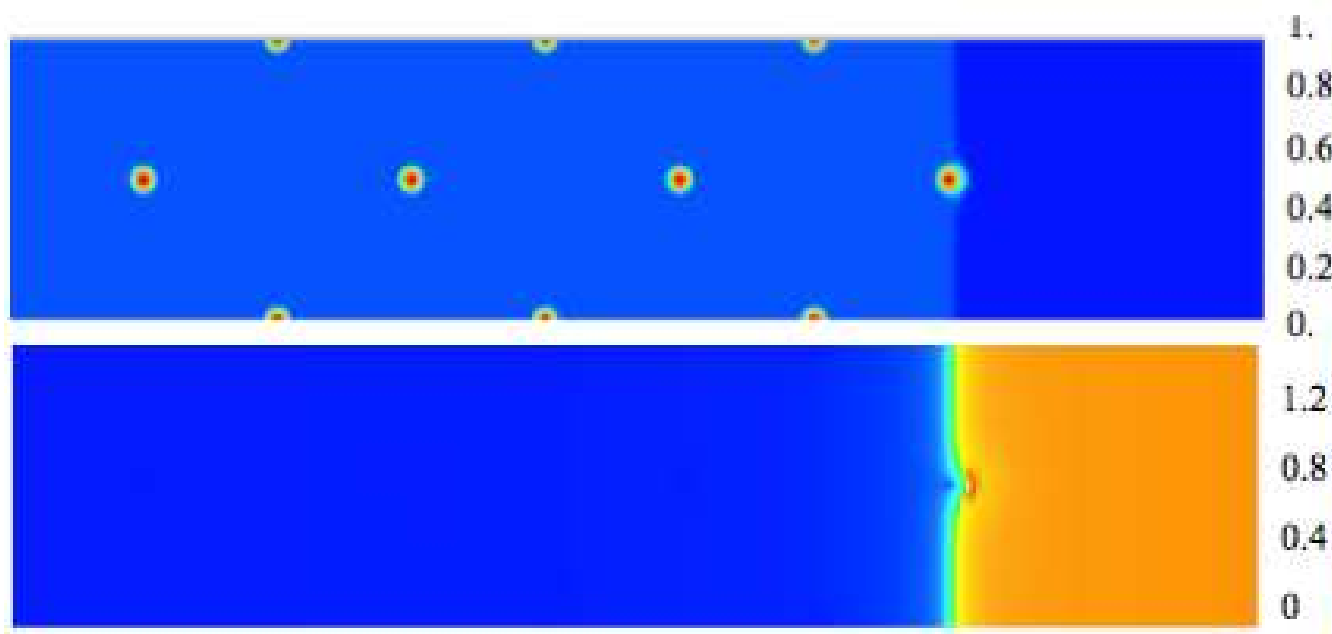

0.

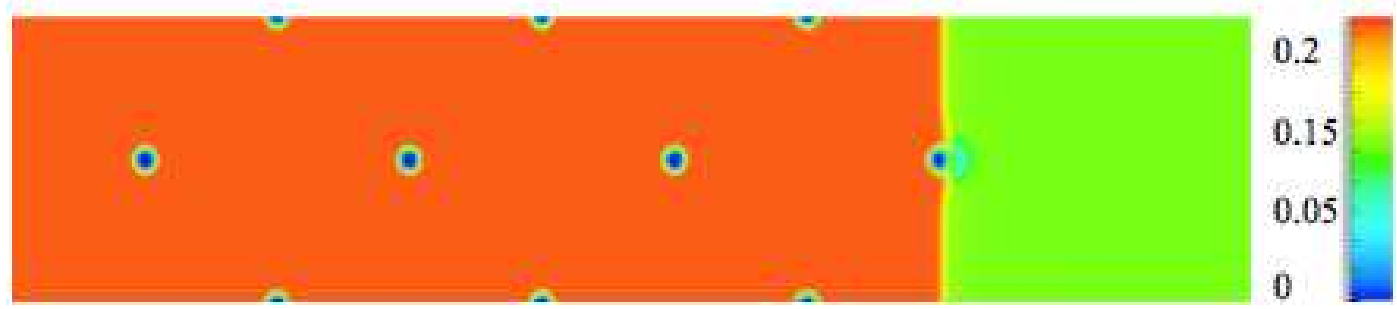

Figure 2: initial fields; a) fuel mass fraction, b) temperature, c) oxygen mass fraction. 
To summarize the conditions of the numerical experiments, in Figure 2 we provide the initial conditions given to the different scalar fields. Figure 2 a recalls the pattern of the initial fuel supply. Figure 2.b illustrates the temperature profile which starts to ignite the lattice, whereas Figure 2.c displays the oxygen initial mass fraction field.

\section{Two-stage spreading}

The plot of $x_{f}$ vs. time presents various shapes as shown in Figure 3, where the flame location is drawn versus time. Combustion spreading develops from the right to the left in the x-direction. This is why the overall slope of the flame position vs. time, i.e. the flame speed, is negative. On the one hand, for large liquid loading, or equivalently for large droplets, the flame front does not propagate monotonously. There are in fact two different stages during the propagation from a large droplet to another: the first one corresponds to vaporization; it is characterized by an apparent front regression, due to the fact that the temperature profile becomes less sharp during the preheating of air and fuel vaporization; therefore, the locus where $\langle T\rangle_{y}\left(x_{f}\right)=0.5$ can appear as regressing. This effect is increased by the fact that gas phase is produced and heated, and the flow that results from the gas expansion pushes this locus back.

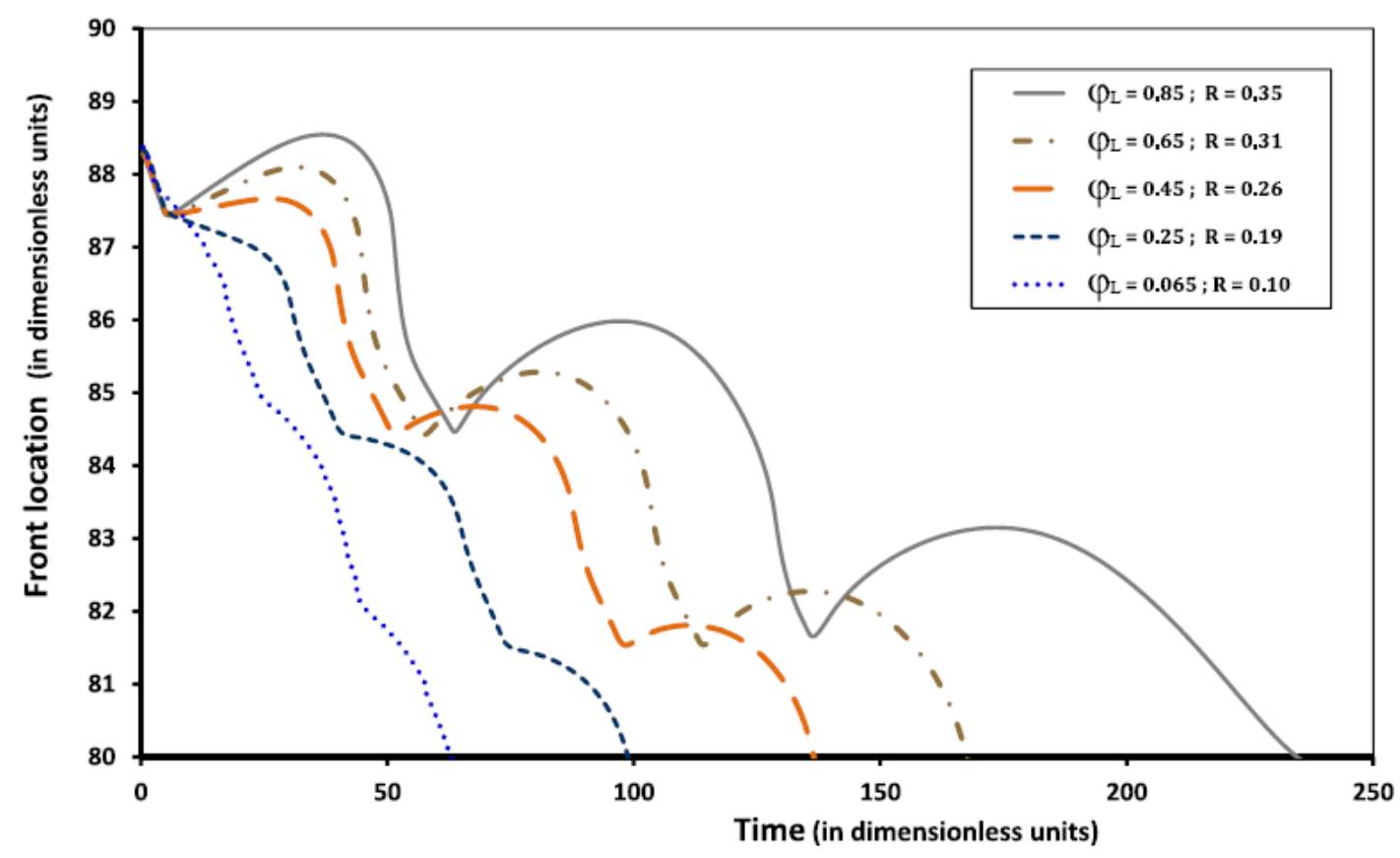

Figure 3: "flame location" vs. time for lattice path $s=6\left(\varphi_{T}=0.85\right)$ and various liquid loading.

The second stage is much shorter in time and corresponds to the reaction stage, which is itself decomposed into two sub-steps: a very rapid propagation of a triple flame-like front which goes around the droplet, followed by the propagation of a slower flame that burns the mixture between the current droplet and the next one. This two-stage propagation remains noticeable as far as the droplet radius is larger than 0.15 (i.e. a small fraction of the stoichiometric gaseous flame thickness). Indeed, this two- 
stage propagation also disappears when the initial pre-mixture increases (i.e for vanishing $\varphi_{L}$ ), as illustrated by the lowest two curves in Fig.3. Those curves correspond to a flammable pre-mixture (their slope is always negative), for which during the vaporization time simultaneously occurs the propagation of a very lean (and therefore slow) flame.

On the other hand, when the droplet radius diminishes (simultaneously with liquid loading), vaporization time becomes shorter, leading us to a flame propagation through a nearly homogeneous medium; two cooperative arguments are advanced for explaining this behaviour: as liquid loading decreases, the pre-mixture increases (since $\varphi_{T}=0.85$ is given), whereas the vaporisation time becomes negligible for smaller and smaller droplets.

For the sake of illustrating the propagation through a large droplet system, we have plotted heat release at various times after ignition in Figure 4. The succession of events can be told as follows. Figure 4.a) provides us with the first instant after ignition. The flame is nearly flat with a very weak heat production, except in the close vicinity of the droplet where the reaction is developing. Figure 4.b) illustrates that the flame skirts round the droplet that vaporizes in the same time. Note the flame is not a strict triple flame, since the rich wing is hardly visible (likely due to high gradients on the rich side). Figure 4.c) shows the diffusion flame that remains behind the front. Combustion spread is now very slow since a weak front of reaction is sustained before the burning droplet. In Figure 4.d) this reaction front becomes even weaker. In the meantime, the next droplet vaporizes. Figure 4.e) illustrates a new stage of intense heat release that corresponds to the combustion of the next droplet, similarly with Figure 4.b). Lastly, Figure 4.f) displays the last instants of life of the diffusion flame, that surrounds the current burning droplet.

Note additionally that the diffusion flame occupies a large "volume". This is due to the large stoichiometric coefficients of heavy alkanes. The above sequence of pictures -with their corresponding instants- corroborates the previous interpretation of Figure 3 about the different stages that characterize the combustion spreading through the lattice. Moreover, the monotonic combustion spreading from the right to the left confirms that there is no front regression (as could have been a possible wrong interpretation of Figure 3).

When the lattice path becomes smaller, the vaporizing fuel pocket (as schematized in Figure 4.b) still remains visible. But, since the flame thickness becomes of the same size as the lattice path, the vaporization of the next droplet is quickly activated. In other words, combustion spread appears rather as a continuous process, close to the propagation in a premixed gaseous medium. The transitional process is investigated in the last paragraph (see Fig. 8).

Note also that the droplets can move during the calculation. As an illustration, the comparison of the heat release presented in Fig.4.b and Fig.4.c (or in Fig.4.e and Fig.4.f) gives a good idea of the droplet motion. The velocity field that drives the droplets results from two cooperative features: fuel droplet vaporization and gas thermal expansion due to flame propagation, the latter feature being classical in any premixed flame propagation. 
a) $t=0$

b) $t=12.5$
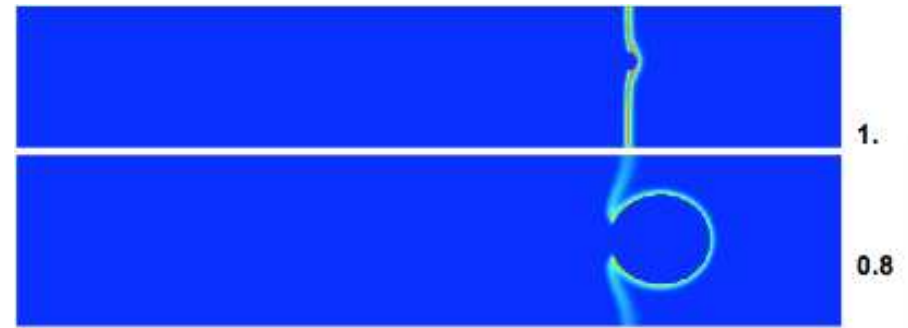

c) $t=19.75$

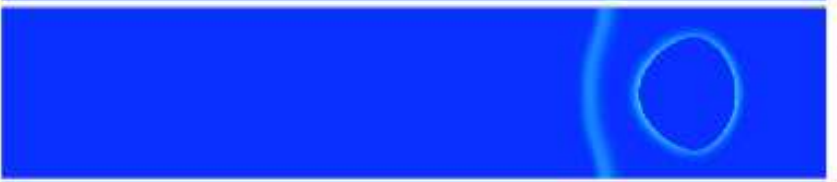

e) $t=37.5$

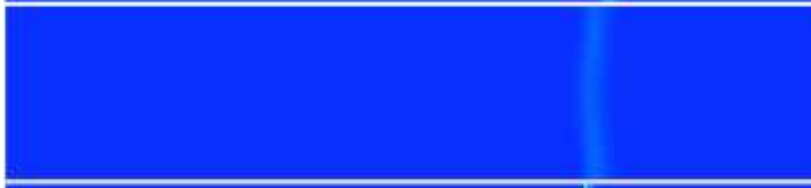

d) $t=25$

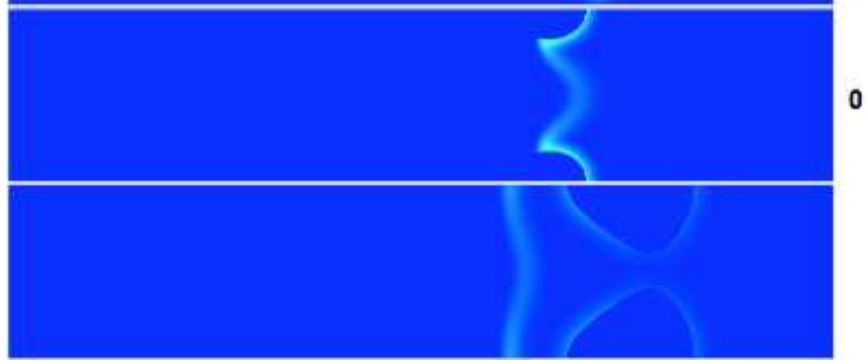

f) $t=46.75$

Figure 4: Snapshots of heat release at various times after ignition

$$
\left(s=6, r_{d}=0.25, \varphi_{G}=0.40, \varphi_{L}=0.45\right) .
$$

\section{Effect of liquid loading}

For fixed overall equivalence ratio $\left(\varphi_{T}=0.85\right)$ and lattice path $s$, increasing the liquid loading corresponds to an enhancement of the droplet radius. Consequently, the vaporization characteristic time being a priori scaled by the square of the radius, the time lag devoted to vaporization is expected to increase. This is illustrated in Figure 5 where the front history is plotted for a large lattice path and various liquid loading (or various corresponding vapour press 


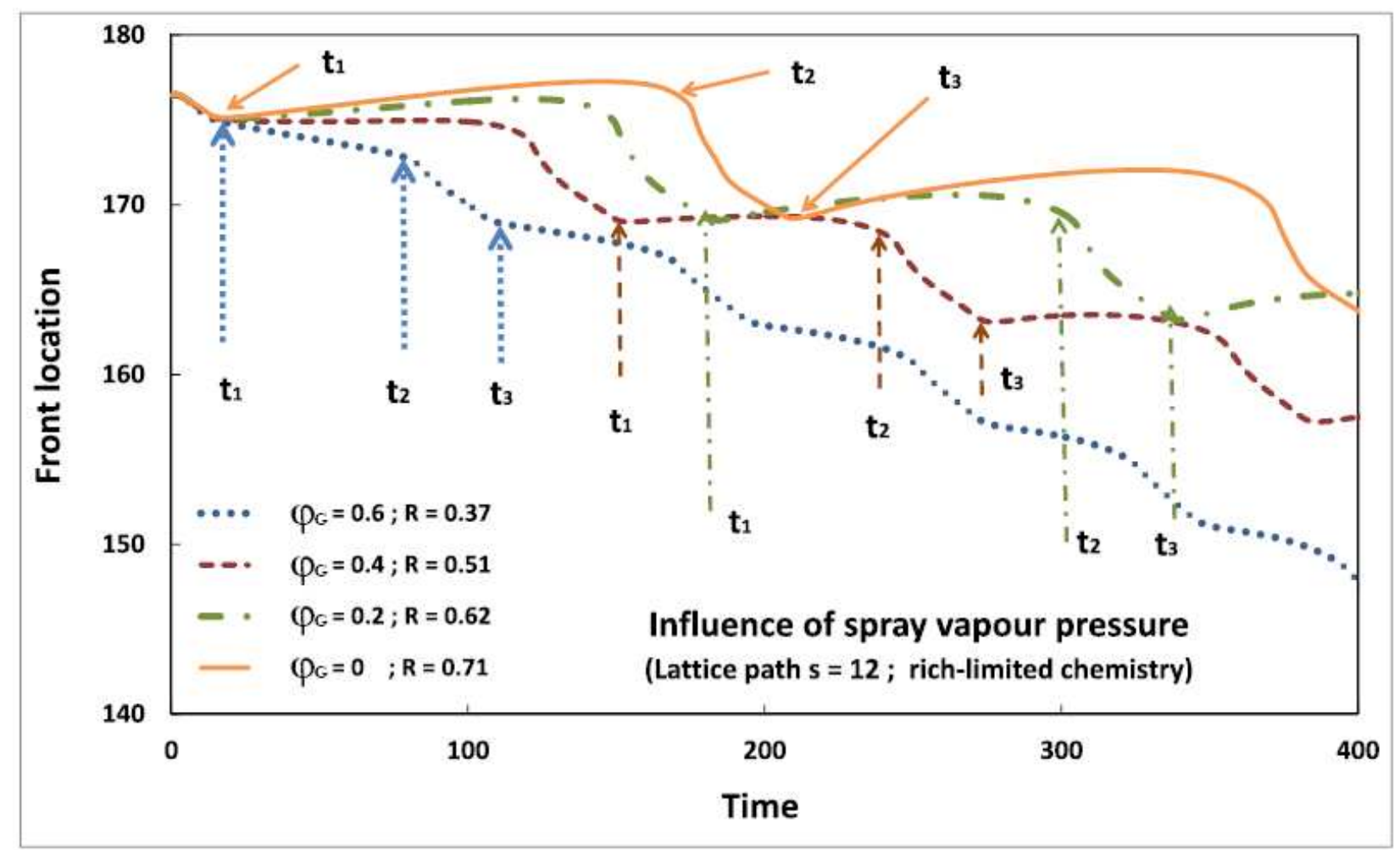

Figure 5: "Front" location vs. time; for $s=12$ and various vapour pressures.

\begin{tabular}{|l|l|l|l|l|l|l|l|}
\hline $\begin{array}{l}s, \text { lattice } \\
\text { path }\end{array}$ & $\varphi_{L}$ & $R$ & $\tau_{v}$ & $\tau_{c}$ & $\frac{\tau_{v}}{R^{2}}$ & $\frac{\tau_{v}}{s^{2} \varphi_{L}}$ & $\frac{\tau_{c}}{s}$ \\
\hline 12 & 0.85 & 0.705 & 164 & 34 & 330 & 1.34 & 2.8 \\
\hline 12 & 0.65 & 0.615 & 118 & 28 & 311 & 1.26 & 2.3 \\
\hline 12 & 0.45 & 0.51 & 77 & 28 & 298 & 1.19 & 2.3 \\
\hline 12 & 0.25 & 0.37 & 51 & 22 & 373 & 1.42 & 1.8 \\
\hline 6 & 0.85 & 0.351 & 52 & 11 & 422 & 1.70 & 1.83 \\
\hline 6 & 0.65 & 0.3 & 41.5 & 11.8 & 461 & 1.77 & 1.97 \\
\hline 6 & 0.45 & 0.255 & 31.3 & 10.6 & 481 & 1.93 & 1.77 \\
\hline 6 & 0.25 & 0.185 & 19.8 & 11 & 578 & 2.20 & 1.83 \\
\hline
\end{tabular}

Table 1: Characteristic times of vaporization and combustion, and their corresponding scalings, for various liquid loadings and lattice paths (with the "Z-corrected 1-step" model).

For $\varphi_{G}=0$ (or $\varphi_{L}=0.85$ ), the flame has to utterly create the mixture permitting its propagation. This is noticeable that the necessary time becomes very large. The different time lags devoted either for vaporization or for reaction are analysed in Table 1, where we report on the 
characteristic times as indicated in Figure 5. Let us define as $\tau_{v}$, the characteristic time for vaporization. As mentioned in Figure 5, its value reads $\tau_{v}=t_{2}-t_{1}$. As for $\tau_{c}$, the combustion time, we define it as $\tau_{c}=t_{3}-t_{2}$. In Table 1 , we only report on $s \geq 6$, because for $s \leq 3$ the different characteristic instants (i.e. $t_{1}, t_{2}$ and $t_{3}$ ) appear less markedly. Accordingly with Table 1 , the characteristic time of the combustion stage only depends on the lattice path (see the fifth column). The combustion step occurs after a vaporization step which also depends on the actual droplet size. Of course, the scaling of the latter time is more or less found in direct ratio to the square of the radius, or equivalently proportional to the product $s^{2} \varphi_{L}$.

To analyse the part played by liquid loading, it must be recalled that combustion must stand far from the droplet, since we are concerned with heavy alkanes. Vaporization must therefore be carried out enough to fill the sphere surrounded by the flame. In other words for globally lean spray, combustion develops intensively only when vaporization is rather complete. This is corroborated by the fact that $\tau_{c}$, the combustion time, does not depend on liquid loading (see the last column of Table 1). On the other hand, for a given lattice path the liquid loading modifies the droplet radius and the initial surrounding gas composition. Therefore, $\tau_{v}$, the vaporization time depends on liquid loading. Accordingly with the penultimate column of Table 1, we found $\tau_{v}$ proportional to liquid loading.

Although we also possess a large set of results for the cases $s=3$ and $s=1.5$, let us observe that Table. 1 does not contain any data from those cases. The reason is the following: as the droplet radius decreases, the determination of the two stages scaled by $\tau_{v}$ and $\tau_{c}$ more and more becomes arbitrary. So that, to derive the scalings of $\tau_{v}$ and $\tau_{c}$, we have decided to exploit the data from large droplets only.

\section{Spray-flame speed}

We have observed that combustion spreads through the lattice with an overall velocity given by the mean slope of the curves drawn in Figures $3 \& 5$. In what follows, this slope is called "spray-flame speed". As mentioned in Introduction, we performed the numerical simulations with the use of two different chemical models. Hereafter, the model that uses $Z$, the mixture fraction, is called " $Z$ corrected 1-step" (and labelled by $Q(z)$ ), while the non-corrected (classical) model is called "Standard 1-step" (and labelled by $Q_{0}$ ), In Figure 6, we plot the numerical spray-flame speed, normalized by the single-phase premixed flame at $\varphi_{G}=\varphi_{T}=0.85$, with respect to droplet radius. In this figure, both chemical models are compared for each case of liquid loading. For a given symbol, the dashed line expresses the results for the standard 1-step model, while the plain line represents the " $\mathrm{Z}$ corrected 1-step model". In the cases drawn here, we observe that the discrepancy remains weak.

Next, we want to use the previous analysis in terms of characteristic vaporization and combustion time scales, in order to interpret the data shown in Figure 6. If we consider a combustion spread through a distance corresponding to the lattice path $S$. As the lattice is face-centred, we meet 2 droplets per lattice period. Therefore, according to Figure 5, we need to spend two vaporization times and two combustion times. Thus, the spray-flame speed reads: 


$$
U_{S F}=\frac{s}{2 \tau_{v}+2 \tau_{c}}
$$

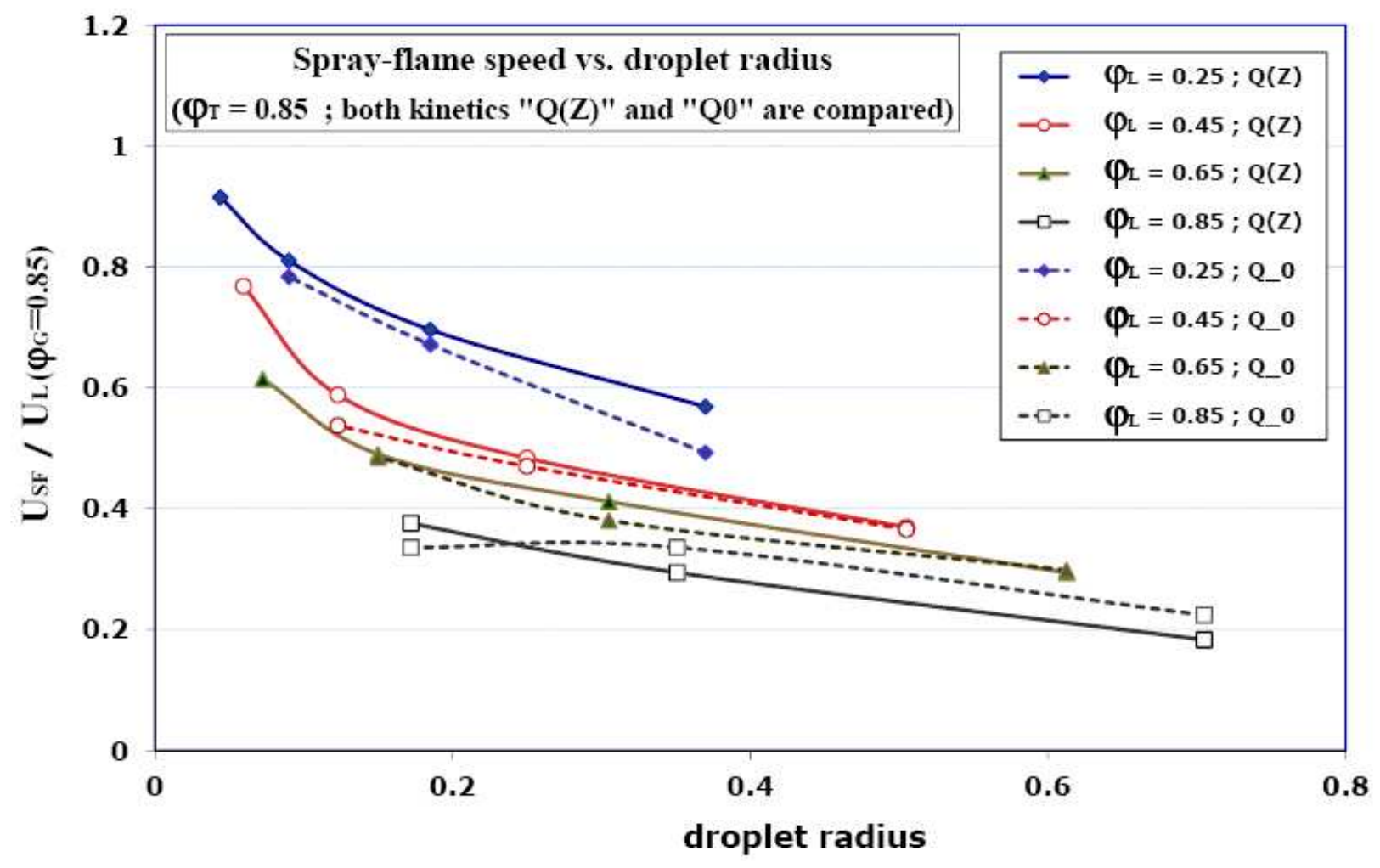

Figure 6: Spray-flame speed (i.e. the combustion spread through the lattice) versus droplet radius for various liquid loading, accordingly with both chemical models

Now, we turn towards Table 1 , where the scales for vaporisation and combustion are analysed. Taking the mean value of the concerned column, we can set $\tau_{v} \approx 1.5 \varphi_{L} s^{2}$ and $\tau_{c} \approx 2 s$. Incorporating those quantities within the spray-flame speed, we simply obtain

$$
U_{S F} \approx \frac{1}{4+3 \varphi_{L} S}
$$

This expression being in terms of the stoichiometric gaseous premixed flame, we are interested in its normalization with respect to the gaseous flame of the same equivalence ratio, denoted by $U_{L}\left(\varphi_{G}=0.85\right)$, i.e. for $\varphi_{G}=\varphi_{T}=0.85$. We then obtain

$$
\frac{U_{S F}}{U_{L}\left(\varphi_{G}=0.85\right)} \approx \frac{13}{8+6 \varphi_{L} s}
$$


Next, we compare the results of this elementary theory with the data obtained from the numerical simulation. This is carried out in Figure 7, where we plot the same velocity data as those of Figure 6 (again normalized with 0.153 , the single-phase flame speed at $\varphi_{G}=\varphi_{T}=0.85$ ) as a function of the factor $\varphi_{L} s$.

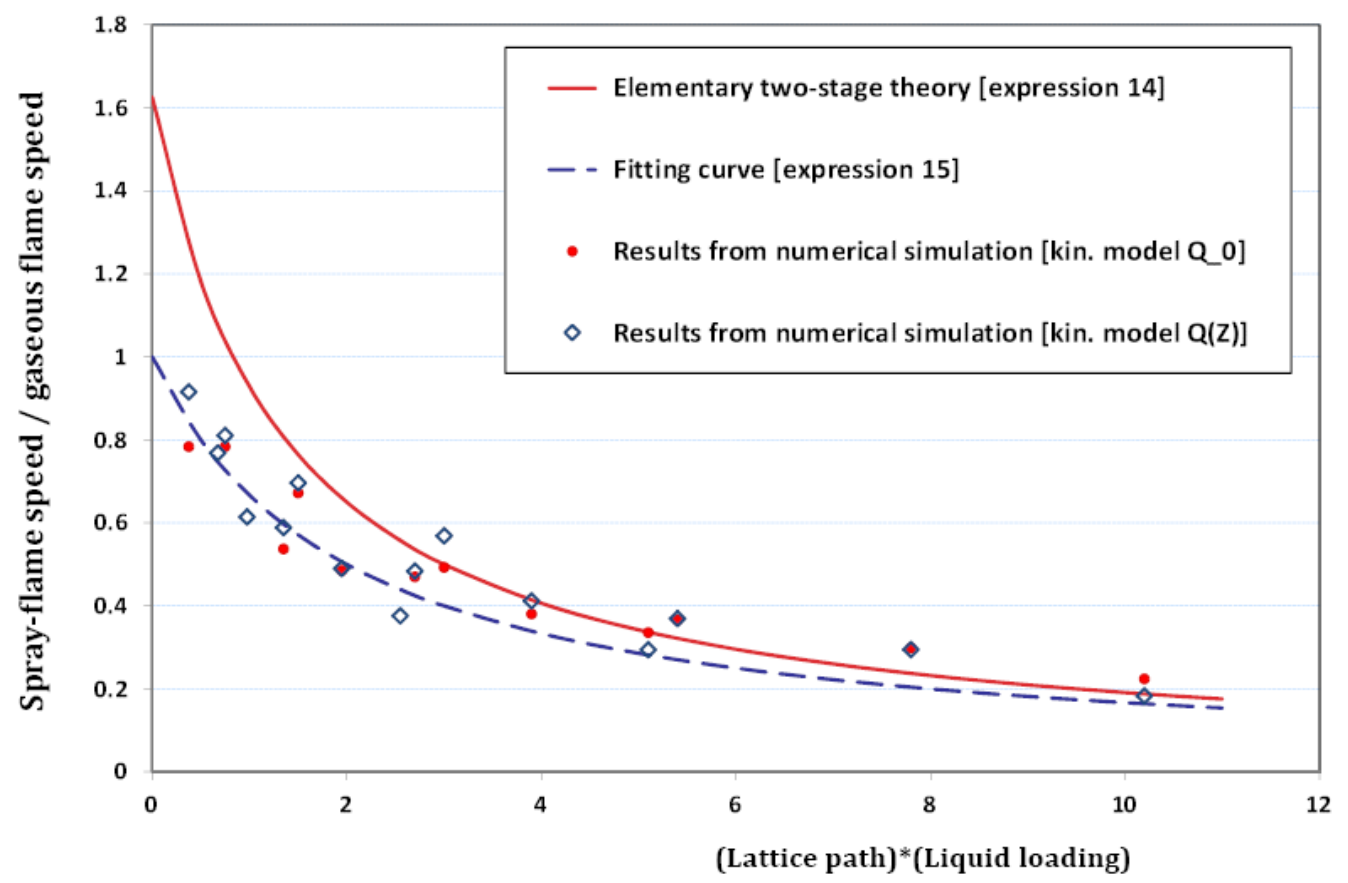

Figure 7: Spray-flame speed (reduced by the single-phase flame speed in a gas mixture of the same overall equivalence ratio) versus the factor $\varphi_{L} s$.

In Figure 7, we perform the plot for the data resulting from both kinetics models. We again observe that the discrepancy between both models remains feeble. Therefore, we conclude that the rough theoretical expression (14) is valid for both chemical models. Note that the cases $s=1.5$ and $s=3$ correspond to the smallest values of the factor $\varphi_{L} S$ in figure 7. It is therefore not surprising that the agreement of the numerical data with our simple model [i.e. formula (14)] is weak in the domain of the small factors $\varphi_{L} s$. Of course, a better agreement would be obtained with the following fit curve

$$
U_{S F} / U_{L}=1 /\left(1+0.5 \varphi_{L} s\right)
$$

which corresponds to the dashed line in Figure 7, while the plain line is related to equation (14). Note additionally that -for a given liquid loading- droplet size are in direct ratio to lattice path. In other words, for large droplets, both equations (14) or (15) retrieve the classical result characterizing the spray combustion regime controlled by vaporization, which behaves as $R^{-1}$ (as observed by Ballal \& Lefebvre 1981 or predicted in Suard et al. 2001). 


\section{Effect of diminishing the lattice path}

As the lattice path shortens, the characteristic time for vaporization (i.e. $\tau_{v} \propto s^{2}$ ) diminishes faster than the characteristic combustion time $\left(\tau_{c} \propto s\right)$. Hence, for small values of $\varphi_{L} s$ (say, $\varphi_{L} s \leq 1$ ), total droplet vaporization and some degree of oxygen diffusion (inside the vapour puff resulting from the droplet vaporization) are carried out before flame spreading. Therefore, when a critical value of $s$ is reached (from above), a rich premixed flame starts to slowly cross the vapour fuel pocket. Then, if the lattice path still decreases (and oxygen diffusion increases), this premixed flame crosses the vapour pocket faster,. Behind the rich premixed flame, a diffusion flame takes place around the puff of fuel vapour. This process for small $s$ is described in Figure 8, where heat release and the corresponding fuel mass fraction are presented at three consecutive times (with a time interval of $\Delta t=0.5)$.
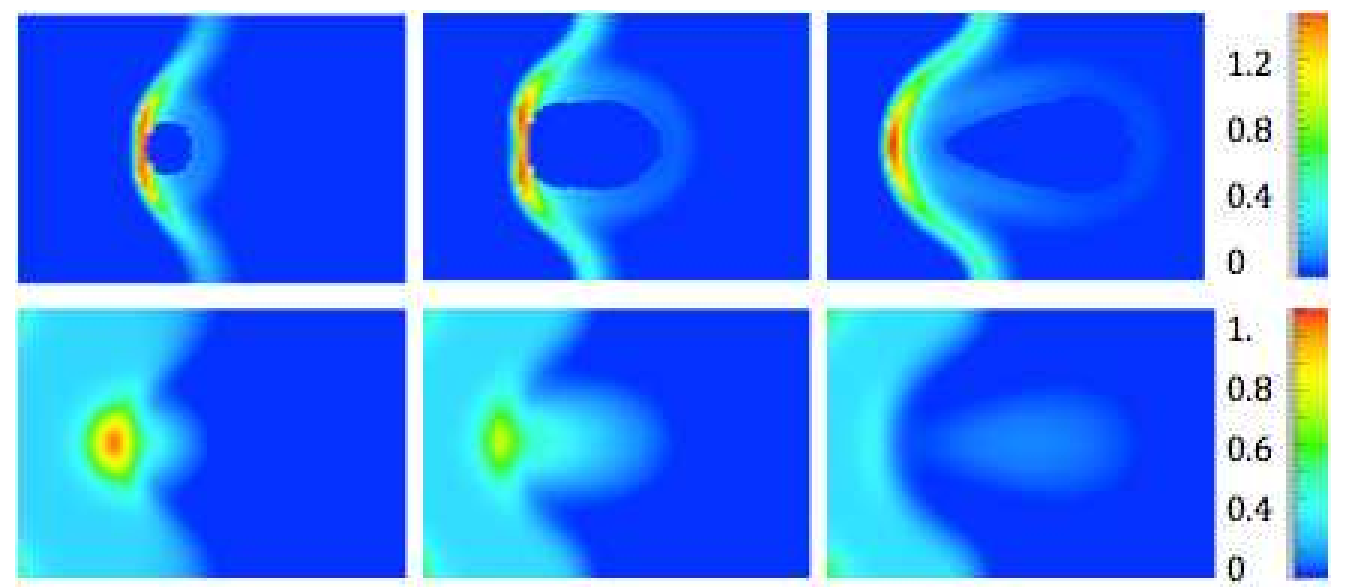

Figure 8. Spreading through a small lattice $\left(s=3 ; \phi_{\mathrm{L}}=0.25\right)$ :

heat release (top) and fuel mass fraction (bottom) at three times separated by $\Delta t=0.5$.

In Figure 8, the puff of vaporized fuel (added with oxygen diffused from its vicinity) is swept by a rich flame, leaving behind a diffusion flame surrounding a hot gas pocket, which still contains an amount of fuel. In the present situation, no clear triple flame occurs since the flame thickness is large enough to vaporize the droplet in the pre-heating zone and to allow diffusion of oxygen towards the fuel puff. The transition to classical (single-phase) premixed flame occurs when this fuel pocket appears negligible for smaller lattice path.

\section{Conclusion}

The present numerical study on lean spray combustion uses a lattice to schematize the droplet deposition. Our results concern the influence of the spray characteristic parameters ("lattice path and liquid loading" or equivalently "droplet radius and liquid loading") on flame dynamics. A particular attention has been paid to focus on large lattice path in comparison with the flame thickness. Additionally, we investigate the transition to premixed flame when the lattice path becomes small.

Two simple chemical schemes with a 1-step exothermic reaction have been used. The first one is associated with the standard Arrhenius law with a reaction heat independent of the equivalence ratio of the mixture to burn. The second one adapts the heat of reaction to the equivalence ratio 
deduced from the field of mixture fraction, with the introduction of a correction applied particularly to rich mixtures.

For both cases of chemical models, we have obtained similar qualitative results in flame structure, as well as quantitative values of spray-flame speed. This indicates that corrections of the triple flame structure on the rich side are probably not very important for flame propagating in a globally lean spray.

Our simulations include a prevaporized fraction of the fuel, which allows the flame propagation from one droplet to the next one through a variable lean pre-mixture. Even for $\varphi_{L}=\varphi_{T}=0.85$ (and $\varphi_{G}=0$ ), no flame extinction has been observed (although combustion spreading was found very slow). We have shown that the combustion spreading for relatively large droplets (compared to the premixed flame thickness) is characterized with two stages: a slow phase corresponding to the partial vaporization of the next droplet until a flammable mixture is carried out, and a faster phase corresponding to the propagation of a triple flame around the droplet.

On the other hand, for smaller droplets, the flame propagates in a regime much closer to a premixed flame, but a premixed flame with a heterogeneous equivalence ratio inside the flame thickness. This regime should deserve a further study because we did not obtain (as visible in Figures 6 and 7) any maximum of the flame speed at non-zero droplet radius. This maximum has, however, been observed in experiments on lean spray-flames in microgravity by Nomura et al. 2000 .

Further studies will turn our attention towards other situations of overall equivalence ratio. Concerning even leaner sprays, we envisage the effects of a random distribution of droplets to explain unexpected flammability of spray, as observed in the experiments of Nunome et al. [2002]. With respect to rich sprays, the experimental contributions are more numerous. The corresponding amount of data should help us to further check the different reduced chemical schemes we have presently used. .

\section{Acknowledgements}

The present work has received the support of the Research Program "Micropesanteur Fondamentale et Appliquée" GDR n²799 CNRS/CNES under the contract CNES/11-5349.

\section{References}

Atzler F., Demoulin F. X., Lawes M., Lee Y., Marquez N., [2006] Burning rates and flame oscillations in globally homogeneous two-phase mixtures (flame speed oscillations in droplet cloud flames), Combustion Science and Technology, Vol. 178, pp. 2177-2198.

Ballal D.R., Lefebvre A.H., Proc. Combust. Inst., Vol. 18 : 321-328 (1981)

Chen C K, Lin T H, . [2012], Streamwise interaction of burning drops , Combustion and Flame , 159 , 19711979

Davis S.G. and Law C.K. [1998], "Linear flame speeds and oxidation kinetics of iso-octane-air and nheptane-air flames", Proc. Combust. Inst., Vol. 22, pp. 521-527.

Denet B., Haldenwang P. [1995], A numerical study of premixed flames Darrieus-Landau instability. Combust. Sci. Techn., Vol. 104, pp. 143-167.

Fernandez-Tarrazo E., Sanchez A.L., Linan A., Williams F.A [2006], A simple one step chemistry model for partially premixed hydrocarbon, Combustion and Flame,. 147, 32-38 .

Garcia-Ybarra P., Nicoli C., Clavin P. [1984], Soret and Dilution Effects on Premixed Flames, Combust. Sci. Tech. 42 , pp. 87-109 
Garrido-Lopez D., Sarkar S. [2005], Effect of imperfect premixing coupled with hydrodynamic instability on flame propagation, Proc. Combust. Inst. ,30, 621-628.

Hara T., Tanoue K. [2006], "Laminar Flame speeds of ethanol, N-Heptane, Iso-octane Air mixtures", International Federation of Automotive Engineering Societies, Student congress paper F2006C40.

Hanai H.; Maruta K., Kobayashi H., Niioka T. [1998], Pulsating flame propagation of PMMA particle cloud in microgravity, Proc. Combust. Inst.. Vol. 27, pp. 2675-2681.

Joulin G., and Mitani J., [1981], Linear stability analysis of two-reactant flames, Combustion and Flame, Vol. 40, pp. 235-246.

Kikuchi M., Wakashima Y., Yoda S., Mikami M. [2005] Numerical study on flame spread of an n-decane droplet array in different temperature environment under microgravity, Proceedings of the Combustion Institute , 30, 2001-2009.

Mikami M., Oyagi H., Kojima N., Kikuchi M., Wakashima Y., Yoda S., [2005] Microgravity experiments on flame spread along fuel-droplet arrays using a new droplet-generation technique, Combustion and Flame $141,241-252$.

Mikami M., Oyagi H., Kojima N., Wakashima Y., Kikuchi M., Yoda S., [2006] Microgravity experiments on flame spread along fuel-droplet arrays at high temperatures, Combustion and Flame 146, 391-406.

Nicoli C., Haldenwang P. and Suard S., [2005] Analysis of pulsating spray flames propagating in lean twophase mixtures with unity Lewis number. Combust. Flame, 143, 299-312 .

Nicoli C., Haldenwang P. and Suard S.,[2007] Effects of substituting fuel spray for fuel gas on flame stability in lean premixtures, Combust. Flame, 149, 295-313.

Nicoli C., Haldenwang P "Analysis of one-step chemistry models for flame propagation in various equivalence ratio premixtures of high alkane-air", in SPEIC10:Towards Sustainable Combustion, Tenerife, June 2010.

Nomura H., Koyama M., Miyamoto H., Ujiie Y., Sato J., Kono M., Yoda S., [2000], Microgravity Experiments of Flame Propagation in Ethanol Droplet-Vapor_Air Mixture, Proc. Combust. Inst. , 28 , 999-1005

Nomura H., Kawasumi I., Ujiie Y., Sato J. [2007], Effects of pressure on flame propagation in a premixture containing fine fuel droplets, Proc. Combust. Inst., 31, 2133-2140.

Nomura H., Takahashi H., Suganuma Y., Kikuchi M., [2013] Droplet ignition behavior in the vicinity of the leading edge of a flame spreading along a fuel droplet array in fuel-vapor/air mixture, Proc. Combust. Inst., Proc. Combust. Inst., Vol. 34 pp. 1593-1600.

Nunome Y., Kato S., Maruta K. Kobayashi H. and Niioka T. [2002], Flame Propagation of n-Decane Spray in Microgravity, Proc. Combust. Inst., 29, 2621-2626.

Oyagi H, Shigeno H, Mikami M, Kojima N [2009], Flame-spread probability and local interactive effects in randomly arranged fuel-droplet arrays in microgravity Combustion and Flame ,156, 763-770

Pichard C., Michou Y. Chauveau C., Gokalp I. (2002), Average Droplet Vaporization Rates in Partially Prevaporised turbulent Spray Flames, Proceedings of the Combustion Institute, Vol. 29, 527-533.

Suard S., Nicoli C., Haldenwang P., [2001], Vaporisation controlled regime of flames propagating in fuellean sprays, J. Phys IV (France), 11, 301-310.

Suard S., Haldenwang P., Nicoli C., [2004], Different Spreading Regimes of Spray Flames, C.R. Acad. Sc. Mécanique (Paris) Vol 332 (5-6), 387-396.

Umemura A, Takamori S [2005], Percolation theory for flame propagation in non- or less-volatile fuel spray: A conceptual analysis to group combustion excitation mechanism, Combustion and Flame 141, 336-349

Wu G., Sirignano W A . [2011], Transient Convective Burning of Interactive Fuel Droplets in Double-Layer Arrays, Combustion and Flame, 158 , 2395-2407. 